\title{
Simultaneous Wireless Information and Power Transfer using Green Communication Based on 5G Wireless Communication
}

\author{
Ahmed Zohair Ibrahim ( $\nabla$ azibrahim@pnu.edu.sa ) \\ Princess Nourah bint Abdulrahman University \\ P Prabu \\ Christ University
}

T Senthilnathan

Christ University

Thangavel Renukadevi

Kongu Engineering College

\section{Research Article}

Keywords: Simultaneous wireless information and power transfer (SWIPT), 5G cellular networks, energy efficiency (EE), internet of things (loT).

Posted Date: November 23rd, 2021

DOl: https://doi.org/10.21203/rs.3.rs-947698/v1

License: (9) This work is licensed under a Creative Commons Attribution 4.0 International License. Read Full License 


\section{Abstract}

Simultaneous wireless information and power transfer (SWIPT) has given new opportunities for dealing with the energe shortage problem in wireless networks.Green transmission for $5 \mathrm{G}$ cellular networks of mobile cloud access networks based on SWIPT is being examined. Considering SWIPT as a future potential solution for increasing the battery life, this technique improves energy efficiency (EE). One of the technologies is wireless communication to transfter the power used to give sufficient resources to energyconstrained networks that have consequences for $5 \mathrm{G}$ and the internet of things (IoT), energy efficiency, co-operative communication and suitable are supported by the SWIPT. To enhance the capacity, data rate improvement, and better performance of quality of services of further networks. In addition to these criteria, it is also our moral responsibility to protect the environment of wireless networks by lowering power usage. As a result, green communication is a critical requirement. We looked at a variety of strategies for power optimization in the impending $5 \mathrm{G}$ network in this article. The utilization of relays and microcells to enhance the network's energy efficiency is the main focus. The many relaying scenarios for next-generation networks have been discussed.

\section{Introduction:}

Wireless information transfer (WIT) over an electromagnetic link is one of these communication technologies. The wireless communication principle is the movement of data from one place to another without the need for a computer in the presence of wires and cable communication. In this situation, distances may be between a few metres and thousands of Km (Jameel et al. 2017). Examples of approaches are developed over time in this domain are satellite communication, infrared communication (IR), and microwave radio. Due to a variety of factors, including high data rate needs, rising energy prices, the ecological impact of carbon, pressure, and ethical responsibility for combating climate change, energy efficiency is becoming a major problem in the telecommunications industry. This has resulted in collaborative research between research and business to develop energy-saving measures, such as the 'green radio' project, the earth project, and others(Amjad et al. 2021).

SWIPT is a wireless communication technology that allows users to access information and collect energy from a signal received, with the recovered energy being used or information relay or processing. WPT (wireless power transfer) and SWIPT have recently attracted a lot of interest since from they open up new oppourtunities for wireless communication systems from the commercial and research (Rajaram et al. 2019). The capacity of the battery only increases 1.5 times a decade and was always a concern of the consumer. With rising numbers of hungry uses of energy in future networks, unlimited access to information and data sharing will take place everywhere and every time. therefore, energy efficiency in wireless communication is necessary to satisfy the user's desire for battery life(Perera et al. 2017).

High power radiated by phones during option tends to damage the user nearby. Therefore, it is all the more necessary to move towards more energy-efficient solutions. The necessity of implementing green communication has been established. Realized all across the world there is a strong emphasis on 
pursuing a holistic approach(Abrol et al. 2018). A method of power optimization the development of new technologies, cell deployment methods, and resource allocation policies are all priorities for nextgeneration systems(Mukhlif et al. 2019). Relays are the key agent of minimizing path loss and thus improving network energy efficiency in the basic design that has been employed in the past to reduce network power consumption(Krikidis et al. 2014).

The work structure as follows; Part 2 defines the highlight of previous work; Part 3 offers the methodology; Part 4 represents the performance of the result and discussion and Part 5 presents the work achieved in conclusion.

\section{Related Work:}

(Wu and Zhang 2019)Presents the lower efficiency of WPT in far-flung areas limits basic rate energy (R-E) efficiency compensation for SWIPT systems. In this paper, propose a novel SWIPT system that is supported by the upcoming IRS technology to resolve this difficulty. IRS achieves a large passive radiation gain that dramatically boosts WPT productivity and hence the SWIPT systems R-E trade-off. The aim is to optimize EHRs received weighted sum power by jointly optimizing the sending procedures of Aps and reflecting changes to the IRs phase, subject to IDR's SINR restrictions. Given that the issue is non-convex, we offer effective strategies to achieve inappropriate answers. We demonstrate that sending information only signals to AP is sufficient, irrespective of their channel achievements, to serve both IDR and EHRs in addition, the simulation findings demonstrate significant performance advantages from our proposed benchmarking approaches.

(He et al. 2018)Evaluates the SWIPT sends wireless nodes to the same information and power of the radio frequency transmission. It might improve the performance of the power-constrained wireless node. SWIPT's current work is focusing on one-hop and two-hop WLAN. This study is simultaneously considered by SWIPPT and MECWN (multi-hops energy-constrained of wireless network, There energy is collected by the receiver node can be used to compensate for data transmission. The energy consumption of the connection transferred with or without SWIPT is assessed with a new routing metric. During path search, the SWIPT routing algorithm for energy-aware assigns data and energy links. Algorithms can harness those tiny sources of energy which are not enough and which have considerably lowered energy use.

(Mowla et al. 2017)Evaluates green communication as the power consumption of communication and information technology, will be another significant aspect of $5 \mathrm{G}$ networks. Service providers require energy-efficient backhauling solutions to enable the all-embracing use of dense small cells. This provides a model of energy-efficient communication for heterogeneous $5 \mathrm{G}$ networks in this research (HetNets). Take into account both access and network backhaul factors. To keep the maximum number of small cells active at cut power consumption during different periods of the day, we create and provide an analytical model to satisfy the service requirement of our consumers. The simulated results show that up to $48 \%$ more power is saved by the suggested green communications strategy than others. 
(Ren et al. 2018) Reducing energy usage while maximizing service quality has become a major challenge in green communications. The processing complexity generated by the huge user terminalsnumber is typically overlooked by conventional network setup methodologies. Formulae a problem optimizing a specific number of operative base stations, user terminal coverage ratio. To optimize the base station'sof ON/OFF status with an estimated rapid coverage, a unique genetic algorithm should be suggested that scaling and selection operators should take into consideration distribution of likelihood of the calculated coverage ratio. The proposed network configuration methodology for green communication was tested and carried out.

\section{Design Methodology:}

\subsection{Green Communication:}

Recent success and demand have been observed by the telecommunications business. There is more than half of the world's population on mobile subscribers. The ICT industry is currently becoming a major global energy consumer. This led academics to explore several techniques to reducing electricity consumption. There are two motivations. Firstly, energy costs are seen as a significant issue in profit estimates by the telecommunication network operators. Secondly, social responsibility for protecting the environment lists a reduction in theICT carbon footprint(Vereecken et al. 2010). In the wireless network's power consumption can be taken from two separate perspectives: base station energy consumption and mobile station electricity consumption. Although the typical mobile phone is decreasing, its functionality is increasing every day, resulting in increased energy usage for new applications as well. Figure. 1 shows the several phases of the life cycle of a mobile device. The most environmental impact is the manufacturing and use of components in the life cycle of a mobile phone. $\mathrm{CO} 2$ output was estimated at $23.8 \mathrm{~kg}$ from the lifecycle of the standard mobile Ericson. Figure.15illustrates the contribution of each phase in the life cycle.

The energy usage at the base station is huge. The whole network's part of the power consumption. The usage of its cycle phase is mainly to increase emissions of carbon(Moberg et al. 2014). Therefore, a holistic approach must be adopted to improve network energy efficiency and move towards green communication. Shrinking and the mobile telephone power consumption discontinuous reception in idle and idle can be used. Mobile phone in the idle DRX mode linked mode. The state is so saving power using no radio resources. CDRX nevertheless wakes up and turns the recipient circuit down. Cyclically saving energy.

\subsection{5g Toward Technologies:}

The world has highly rapid developments and technological revolutions, notably in computers and wireless networks, from $2 \mathrm{G}$ to $4 \mathrm{G}$. More energy economy, bandwidth, and decreased latency were the major reasons. Thus, $4 \mathrm{G}$ is the first mobile wideband standard, but $3 \mathrm{G}$ has been designed for voice 
combined with some data and multimedia considerations, while $2 \mathrm{G}$ is intended to be the first standard for digital mobile voice communication with increased coverage. The data rate from $64 \mathrm{kbps}$ in $2 \mathrm{G}$ to $2 \mathrm{Mbps}$ in $3 \mathrm{G}$ has improved, and from 50 to $100 \mathrm{Mbps}$ in $4 \mathrm{G}$ has improved(Bega et al. 2017). In addition to improving the mobile network speed data transmission, it is projected that $5 \mathrm{G}$ will improve network connection, scalability, and energy efficiency. By 2020, 50 billion devices are expected to be connected to the worldwide IP network and this appears to be a challenge. Therefore, $5 \mathrm{G}$ describes high output, high reliability, low latency, powerful mobile technology, and greater scalability as the main elements. This allows end-users to enjoy smooth connectivity.

The future society, however, will be related, loT, in conjunction with smart, integrated sensor systems and home sensor networks, will revolutionize people's lives. It also enhances energy efficiency, use of spectrum, and cost. It is also scalable to meet the increasing number of linked devices. The overall technological objective of the current network is to provide an idea that supports a high latency, increasing data volume, machine-to-machine communications and high energy, and spectral effectiveness, given the vision of the entire communicating globe.

\subsection{Techniques Of Energy Efficient:}

The equivalent necessity for energy consumption optimization is likewise increasing weith the advancement of communication technology. According to a study, shows that mobile carriers are among the highest consumers of energy and that their consumption is expanding quite rapidly, in particular with the implementation of $4 G$ technology. Both energy efficiency from the point of view of the user. It is crucial in wireless communication. The energy need efficient transmission comes from the restricted energy. Ad hoc networks where wireless devices are available are powered by batteries so that energy consumption is required mimized. To make the network energy efficient, we have different options build energy-efficient designs or make use of energy-efficient radio technology or achieve resource management energy efficiency. The approaches in the table were further examined and we focus on power optimization use relays and their $5 \mathrm{G}$ network integration(Mahapatra et al. 2015).

This strategy could be most useful for the sensor data or for the loT of objects technologies used to load the access point by using control signals. With MIMO, small cells, and mm waves, future networks will overcome their direction loss issues. The element utilized is a rectenna that transforms microwave radiation into direct current. The signal received is split into two orthogonal signals. The current communication system is modified by the SWIPT. Three situations can be used for SWIPT:

- Close to field state:The power of the inductor is transmitted or condenser connection, with transmission range of $1 \mathrm{~m}$ up to 10 watts.

- Far-field State: Power is delivered utilized the beaming of power directive with a orderup to-MW antenna with multi-meter range. 
- Low power Far Field: Power is shifted to microwatts with the RF power scavenging range of several kilometers.

Figure 3 shows simultaneous information and transmission of power between mobile users and a MIMO basis station. We now focus on the far-flung scenario. The received power signal shall therefore be divided into two signals, one for collecting energy and the other for decoding information.

\subsubsection{SWIPT:}

The collection of energy from the environment and the transformation of energy into electricity are attractive ways to operate wireless communication networks. Although the energy required to operate the system does not decrease directly by this approach, wireless networks can clean energy sources and power renewable. Two major energy collection kinds, environmental and radio frequencies, have arisen so far within the context of wireless communication. Radio-frequency energy collection also offers an attractive opportunity to reduce the allocation of wireless energy sources. The concept combines energy collection with wireless technology for the transmission of power(Rostampoor, Razavizadeh, and Lee 2017). It allows the sharing of energy between nodes of network, extending the nodes life when the battery power is low. Simultaneous wireless communication and power transmission are the basis of this technology by the energy superimposition signal on ordinary communications signals (SWIPT). The technique is mostly connected to 5 chains and networks (MISO, MIMO, Relay Channel network, Cooperative of Networks, and Allocation of Resource), as demonstrated in figure.3. from a comprehensive critical evaluation conducted on SWIPT for over 90 articles. The main critical review table is then divided into five tables according to SWIPT.

The technology can be used for the wireless loading of power restricted relay nodes. In $5 \mathrm{G}$ networks, more wandering RF signals will be available with the arrival of massive MIMO technology, which can be used on the collecting power relay(Xu et al. 2018). In the near field scenario, SWIPT as mentioned is more successful. Since it is envisaged that the $5 \mathrm{G}$ networks would include Femtocells within buildings, a wireless transmission system can be readily integrated for mobile indoor equipment. The main aim is to keep emissions below the established criteria in this area.

\subsubsection{Millimeter Waves:}

One of the most promising $5 \mathrm{G}$ technology is expected to be millimeter waves. Bandwidth allocation for quick video and multimedia content transmission is supposed to solve the problem. As the wavelength of the mileage is quite little, it uses both transmission and reception spatial multiplexing techniques. In the millimeter range, massive MIMO will play an important role(Hao et al. 2019). The transmission node can transmit a direct signal to the appropriate receiver using suitable signal processing techniques. The steerable array antennas are therefore employed to achieve a high data rate and capacity within a 
millimeter range. The use of radiation focus beam shaping enhances the communication range as well as the energy consumption is projected to decrease. The employment of a directional transmission antenna array between the base and a mobile systems lower signal interference, reducing energy efficiency(Wagih, Weddell, and Beeby 2019). When a direct connection to eliminate interference is created, better data speeds can be attained for certain levels of transmission energy. This will increase the unot energy output in this scenario and hence improve energy efficiency.

\subsubsection{Small Cell:}

Small cells is an umbrella phrase employed in licensed spectrum operating operators managed, lowpowered and low-cost stations. They can be used intensively to achieve high data rates. Small cell sizes can vary based on the size: Femtocells (up to 100m); Pico cells (up to $200 \mathrm{~m}$ ); Macro Cells (up to $500 \mathrm{~m}$ ). A comparison of cell installations by area and capacity may be seen in the graph in Fig. 5 . Small cells can have a central baseline or remote wired or wireless radio heads using the core network(Lohani, Hossain, and Bhargava 2016). They also lowerthe user distance from the Base Station (BS) and reduce transmission capacity required to overcome the pathless, particularly in the indoor setting. There is a small cell connection point on buildings and the base station communicates. In the building, the mobile stations must only be transmitted to the SCA and thereby decrease both the load and energy requirements, not the far-flung base station. Small cell deployment requires few changes to the present standard and can save a lot of battery use from the user.

\subsubsection{Massive Mimo:}

A MIMO version is proposed in $5 \mathrm{G}$ networks, in which a huge antennas numberin the BS known as the Massive MIMO are used. The base station can therefore simultaneously communicate with several users in the same frequency range using this technology, enabling high multiplexing and array gain. Not only spectrum efficiency, but energy efficiency as well are massive MIMO technology. It is discovered that the antennas numberin the BS reduces the power transmitting so that the small data rate as a single antenna system with channel status data is known. In addition to the power scaling rule, substantial emphasis has been paid to techniques to improve energy efficiency in massive MIMO systems(Yang et al. 2020). Increasing spectral efficiency and complete channel information have demonstrated energy-efficiency decreases. With inadequate state information on channels, energy efficiency in the low-power region increases with spectral efficiency and declines in the high-power zone. It is evident that with massive MIMO many antennas consume tremendous power, which leads to considerable energy efficiency reductions.

\subsubsection{Relay:}


The essential principle used in transmission is that moving stations can receive a signal in a relay-aided network both from the base station (BS) and from a relay node (RN) that delivers a better signal force that varies mostly on their distance(Li and Yang 2019). It varies from the normal wireless network in which every mobile sation talks to a base station directly. This makes it different. Relay divided longer routes into shorter ones by LOS communication and reduce total loss of route the power required for the transmission is thereby reduced. The low power of transmission also lowers interference. Using relays, as already discussed, is a better approach to increase the energy efficiency of a wireless network. Consideration of the benefits of employing relays transmission delay and use of electricity. Using realys, it is shown that CDMA cellular networks may cut power usage. The rsults show that more power is aved, the higher the track loss exponent. The balance in the channel if the AWGN relay between energy and adat rate. The hop number impact user position and energy distribution on energy is also shown efficiency. The relay encounters decreasing conditions that are separate from the direct path between source and source destination.

We have thought of one techniques of relaying so far. Recently, solutions were applied based on two-way relay network coding. There are three basic reay system: decode and forward, compress and forward, and enhance and advance. In the pioneer article, cover and El-Gamal initially developed DF and Af techniques in (Najafi et al. 2015)and above the bottom limits of a general relay.

- Decode and forward(DF):

The ralays decode the source message in one block in this relaying method are shown in figure. 6 and send the encoded data in a block instead of forwarding individual packets. This reduces transmissions and hence reduces energy consumption

- Amplify and forwards (AF):

The relay will send an amplified version of the signal received in the last time-slot in this relaying method as shown in figure.7.

- Compress and Forward (CF):

In this transmission system, the relay quantizes the signal received in a single block and in the following block transmits in the folloeing block transmits the coded from of the received signal.

AF requires substantially less time when compared to $D F$ and $C F$, since the relay knot works time slot by time-slot. Also, AF takes far less power than decoding. On the relay side, the quantizing procedure is done. Networking the OFDMA-based system for coding is proposed(Salem et al. 2010). Relay networks termed "XOR-CD" for considerable utilization.In-band/out-band relaying, as well as transparent and nontransparent user connections have been detailed relay standard. But numerous challenges like deployment of full duplex relays, D2D relaying, communication with mobile relays are remaining for implementation in next generation networks(Deng et al. 2015). Various devices and various applications 
should be supported by next-generation networks. For different application, different relay selection policies are proposed. The following are:

- High mobility scenarios are provided with a reduced channel estimate overhead policy.

- A policy to minimize delay is adopted for a critical delay application.

- A power-efficient communication is low-energy policy.

\section{Result And Discussion:}

In this section, discuss the results attained from the SWIPT on green communication based on $5 \mathrm{G}$ wireless communication. A comparison of relay achieves higher than the target data rates and so improves the system capacity in figure.8. A component of the relay battery is resistant to the attacker's network intrusion attack. It's also going to be consumed. As a result, due to the energy limitation, it cannot relay data for a long time. the data rate reached after the intrusion by the relay action may or may not exceed the data threshold.

Figure.9indicates energy efficiency if the iteration number is SWIPT and non-SWIPT from 0 to 20 . This figure indicates each small base station (SBSs) has a maximum capacity of $30 \mathrm{dBm}$, each SBSs is set to 10 and each SBS has a maximum capacity of 6 . The system energy efficiency (EE) can coverage in 8 iterations, as shown in this figure. The EE of the SWIPT system is around 2.3 to $107 \mathrm{bps} / \mathrm{joule}$, while the energy efficiency of the joule scheme is roughly 1.6 to $107 \mathrm{bps} /$ joule without SWIPT. The scheme with the energy collection units may collect energy when transmitted so that energy consumption can be greatly reduced. The system performance can improve by 1.5 times compared with the case without SWIPT.

Figure.10illustrated the EE system where the user's number per small cell is 4 to 20 and 20. Accordingly 20,30 , and 50 for small cell numbers. Each SBS has a maximum output of $23 \mathrm{dBm}$. In the figure, the system EE increases accordingly increases the number of SBSs and users of SBSs. If the number for SBS is 50 , the EE is more than 20 and 30 for the SBS numbers. The EE of 50 SBSs is almost twice as high as 30 and 2.5 times as high as 20 SBSs. The more SBSs and users can find the greater the system performance.

The next generation of networks should meet user's expectations and provide a green communication solution. New technologies like SWIPT, MIMO, mm wave and the continued application of small cells and relay will pose new challenges to research in future generation networks. A full duplex relay channel EE of the huge MIMO network has got to be studied. To eliminate the effect of interference, the EE of large MIMO in many cell scenarios has ti be studied. Additional studies must also be done in order to integrate base station sleep modes efficiently to preserve maximum power. Small cells necessitate a research of their power allocation techniques for the base station and its impact on network energy efficiency. The probable future work in this area will be the impact of several antennas at the base station and on the joint resource and power assignment. The effect multiple baseline antennas and joint power and resource 
attribution in this respect is the prospective future work. The compromise between hardware use and energy savings of the network with massive beam-forming MIMO. The range of millimeters must also be studied together with the network's total energy efficiency.

\section{Conclusion:}

This study explores in the next generation networks the growing requirement of energy efficiency. We evaluated the developments in wireless communications over the last decade that have shown a change for the next generation network towards green communication. There was also a discussion about the need of selecting a suitable EE metric. An EE measurement model with the relay selection use and a comparison of several EE in relay-based contexts were also provided. The suggested design has also included EE techniques like Massive MIMO, C-ran, and SWIPT. The focus is mostly on the usage of relays and their significance in improving the energy efficiency in the next generation networks and small cells. The research also discusses the influence on the carbon footprint of mobile life cycle analysis for green communication purposes.

\section{Declarations}

\section{Ethical Compliance}

Not applicable

\section{Conflicts of Interest}

There is no conflict of interest

\section{Funding}

There is no funding

\section{Acknowledgments}

All authors have seen the manuscript and approved to submit it to the journal.

\section{Informed Consent}

All authors have seen the manuscript and approved to submit it to the journal.

\section{Author contributions}

All authors have seen the manuscript and approved to submit it to the journal.

\section{References}


1. Abrol, Akshita, Rakesh Kumar Jha, Sanjeev Jain, and Preetam Kumar. 2018. "Joint Power Allocation and Relay Selection Strategy for 5G Network: A Step towards Green Communication."

Telecommunication Systems 68 (2): 201-15.

2. Amjad, Maliha, Omer Chughtai, Muhammad Naeem, and Waleed Ejaz. 2021. "SWIPT-Assisted Energy Efficiency Optimization in 5G/B5G Cooperative loT Network." Energies 14 (9): 2515. https://doi.org/10.3390/en14092515.

3. Bega, Dario, Marco Gramaglia, Carlos Jesus Bernardos Cano, Albert Banchs, and Xavier Costa-Perez. 2017. "Toward the Network of the Future: From Enabling Technologies to $5 \mathrm{G}$ Concepts."

Transactions on Emerging Telecommunications Technologies 28 (8): e3205.

4. Deng, Junquan, Alexis A Dowhuszko, Ragnar Freij, and Olav Tirkkonen. 2015. "Relay Selection and Resource Allocation for D2D-Relaying under Uplink Cellular Power Control." In 2015 IEEE Globecom Workshops (GC Wkshps), 1-6. IEEE.

5. Hao, Wanming, Gangcan Sun, Fuhui Zhou, De Mi, Jia Shi, Pei Xiao, and Victor CM Leung. 2019. "Energy-Efficient Hybrid Precoding Design for Integrated Multicast-Unicast Millimeter Wave Communications with SWIPT." IEEE Transactions on Vehicular Technology 68 (11): 10956-68.

6. He, Shiming, Kun Xie, Weiwei Chen, Dafang Zhang, and Jigang Wen. 2018. “Energy-Aware Routing for SWIPT in Multi-Hop Energy-Constrained Wireless Network." IEEE Access 6: 17996-8.

7. Jameel, Furqan, Faisal, M. Asif Ali Haider, and Amir Aziz Butt. 2017. "A Technical Review of Simultaneous Wireless Information and Power Transfer (SWIPT)." In 2017 International Symposium on Recent Advances in Electrical Engineering (RAEE), 1-6. Islamabad: IEEE. https://doi.org/10.1109/RAEE.2017.8246039.

8. Krikidis, loannis, Stelios Timotheou, Symeon Nikolaou, Gan Zheng, Derrick Wing Kwan Ng, and Robert Schober. 2014. "Simultaneous Wireless Information and Power Transfer in Modern Communication Systems." IEEE Communications Magazine 52 (11): 104-10.

9. Li, Quanzhong, and Liang Yang. 2019. "Robust Optimization for Energy Efficiency in MIMO Two-Way Relay Networks with SWIPT.” IEEE Systems Journal 14 (1): 196-207.

10. Lohani, Sudha, Ekram Hossain, and Vijay K Bhargava. 2016. “On Downlink Resource Allocation for SWIPT in Small Cells in a Two-Tier HetNet." IEEE Transactions on Wireless Communications 15 (11): 7709-24.

11. Mahapatra, Rajarshi, Yogesh Nijsure, Georges Kaddoum, Naveed Ul Hassan, and Chau Yuen. 2015. "Energy Efficiency Tradeoff Mechanism towards Wireless Green Communication: A Survey." IEEE Communications Surveys \& Tutorials 18 (1): 686-705.

12. Moberg, \AAsa, Clara Borggren, Christine Ambell, Göran Finnveden, Fredrik Guldbrandsson, Anna Bondesson, Jens Malmodin, and Pernilla Bergmark. 2014. "Simplifying a Life Cycle Assessment of a Mobile Phone." The International Journal of Life Cycle Assessment 19 (5): 979-93.

13. Mowla, Md Munjure, Iftekhar Ahmad, Daryoush Habibi, and Quoc Viet Phung. 2017. "A Green Communication Model for $5 \mathrm{G}$ Systems." IEEE Transactions on Green Communications and Networking 1 (3): 264-80. 
14. Mukhlif, Fadhil, Kamarul Ariffin Bin Noordin, Ali Mohammed Mansoor, and Zarinah Mohd Kasirun. 2019. "Green Transmission for C-RAN Based on SWIPT in 5G: A Review." Wireless Networks 25 (5): 2621-49. https://doi.org/10.1007/s11276-018-1718-z.

15. Najafi, Marzieh, Mehrdad Ardebilipour, Ehsan Soleimani-Nasab, and Saeed Vahidian. 2015. "MultiHop Cooperative Communication Technique for Cognitive DF and AF Relay Networks." Wireless Personal Communications 83 (4): 3209-21.

16. Perera, Tharindu D Ponnimbaduge, Dushantha Nalin K Jayakody, Shree Krishna Sharma, Symeon Chatzinotas, and Jun Li. 2017. "Simultaneous Wireless Information and Power Transfer (SWIPT): Recent Advances and Future Challenges." IEEE Communications Surveys \& Tutorials 20 (1): 264302.

17. Rajaram, Akashkumar, Rabia Khan, Selvakumar Tharranetharan, Dushantha Jayakody, Rui Dinis, and Stefan Panic. 2019. "Novel SWIPT Schemes for 5 G Wireless Networks." Sensors 19 (5): 1169. https://doi.org/10.3390/s19051169.

18. Ren, Yebing, Wei Liu, Jiangbo Dong, Haobin Wang, Yaxi Liu, and Huangfu Wei. 2018. "Genetic Algorithm for Base Station on/off Optimization with Fast Coverage Estimation and Probability Scaling for Green Communications." In International Conference On Signal And Information Processing, Networking And Computers, 78-88. Springer.

19. Rostampoor, Javane, S Mohammad Razavizadeh, and Inkyu Lee. 2017. "Energy Efficient Precoding Design for SWIPT in MIMO Two-Way Relay Networks." IEEE Transactions on Vehicular Technology 66 (9): 7888-96.

20. Salem, Mohamed, Abdulkareem Adinoyi, Mahmudur Rahman, Halim Yanikomeroglu, David Falconer, Young-Doo Kim, Eungsun Kim, and Yoon-Chae Cheong. 2010. "An Overview of Radio Resource Management in Relay-Enhanced OFDMA-Based Networks." IEEE Communications Surveys \& Tutorials 12 (3): 422-38.

21. Vereecken, Willem, Ward Van Heddeghem, Didier Colle, Mario Pickavet, and Piet Demeester. 2010. "Overall ICT Footprint and Green Communication Technologies." In 2010 4th International Symposium on Communications, Control and Signal Processing (ISCCSP), 1-6. IEEE.

22. Wagih, Mahmoud, Alex S Weddell, and Steve Beeby. 2019. "Millimeter-Wave Textile Antenna for onBody RF Energy Harvesting in Future 5G Networks." In 2019 IEEE Wireless Power Transfer Conference (WPTC), 245-48. IEEE.

23. Wu, Qingqing, and Rui Zhang. 2019. "Weighted Sum Power Maximization for Intelligent Reflecting Surface Aided SWIPT." IEEE Wireless Communications Letters 9 (5): 586-90.

24. Xu, Kui, Zhexian Shen, Yurong Wang, and Xiaochen Xia. 2018. "Beam-Domain SWIPT in Massive MIMO System with Energy-Constrained Terminals." IET Communications 12 (15): 1900-1909.

25. Yang, Xiaoxia, Zhengqiang Wang, Xiaoyu Wan, and Zifu Fan. 2020. "Secure Energy-Efficient Resource Allocation Algorithm of Massive MIMO System with SWIPT." Electronics 9 (1): 26.

\section{Figures}




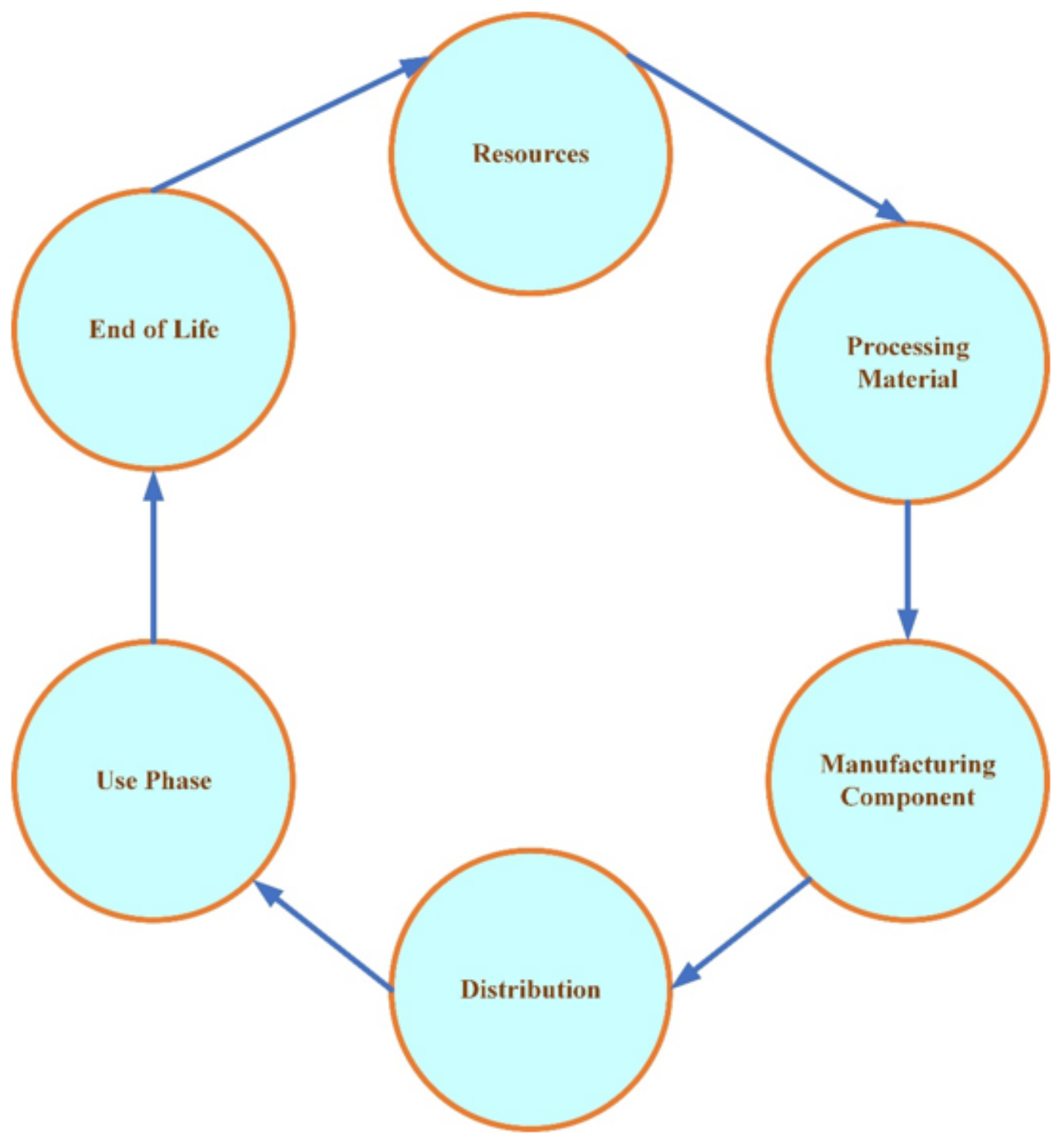

Figure 1

The life cycle of mobile phone assessment. 


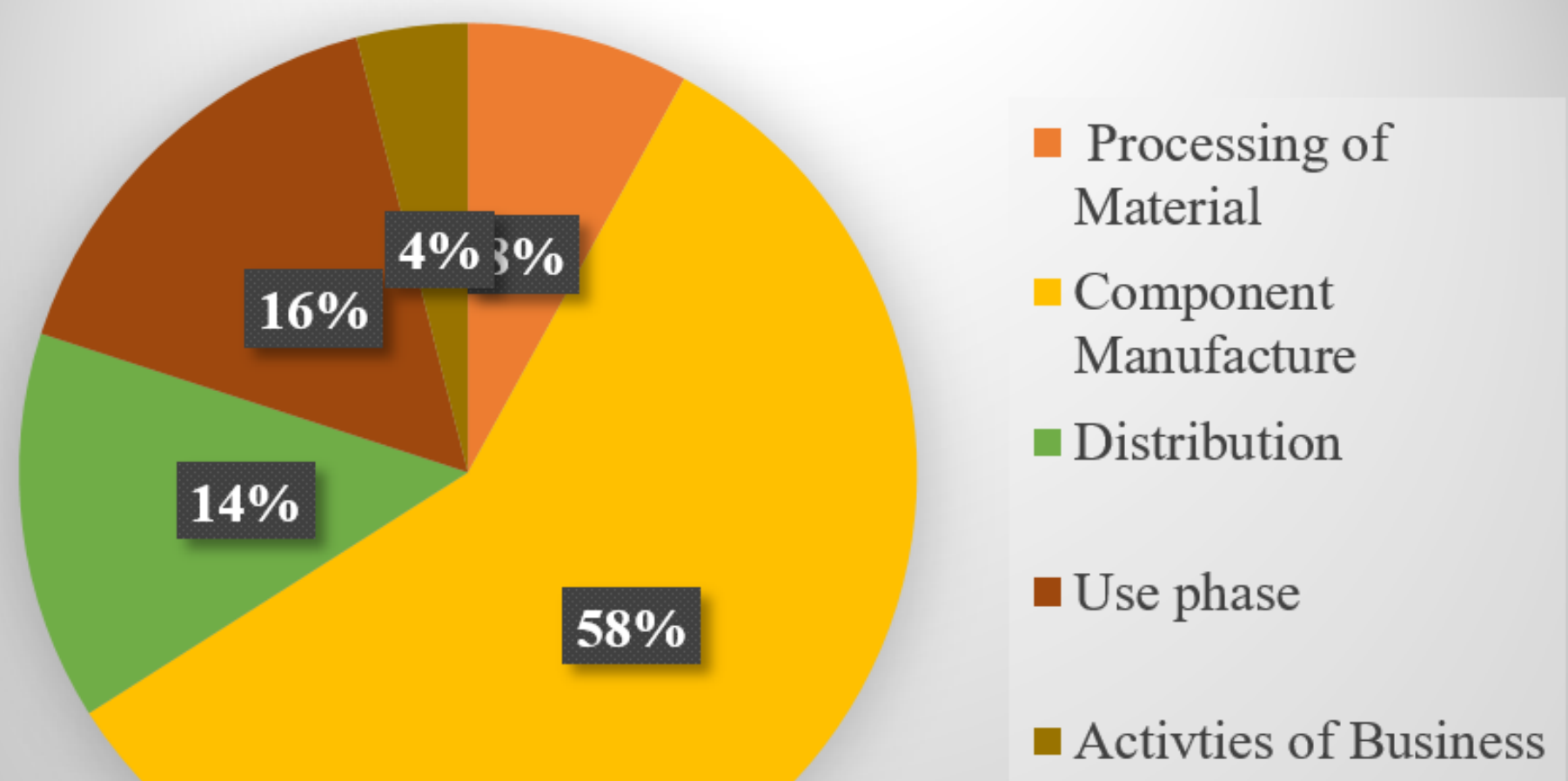

Figure 2

Lifecycle of mobile phone carbon emission 


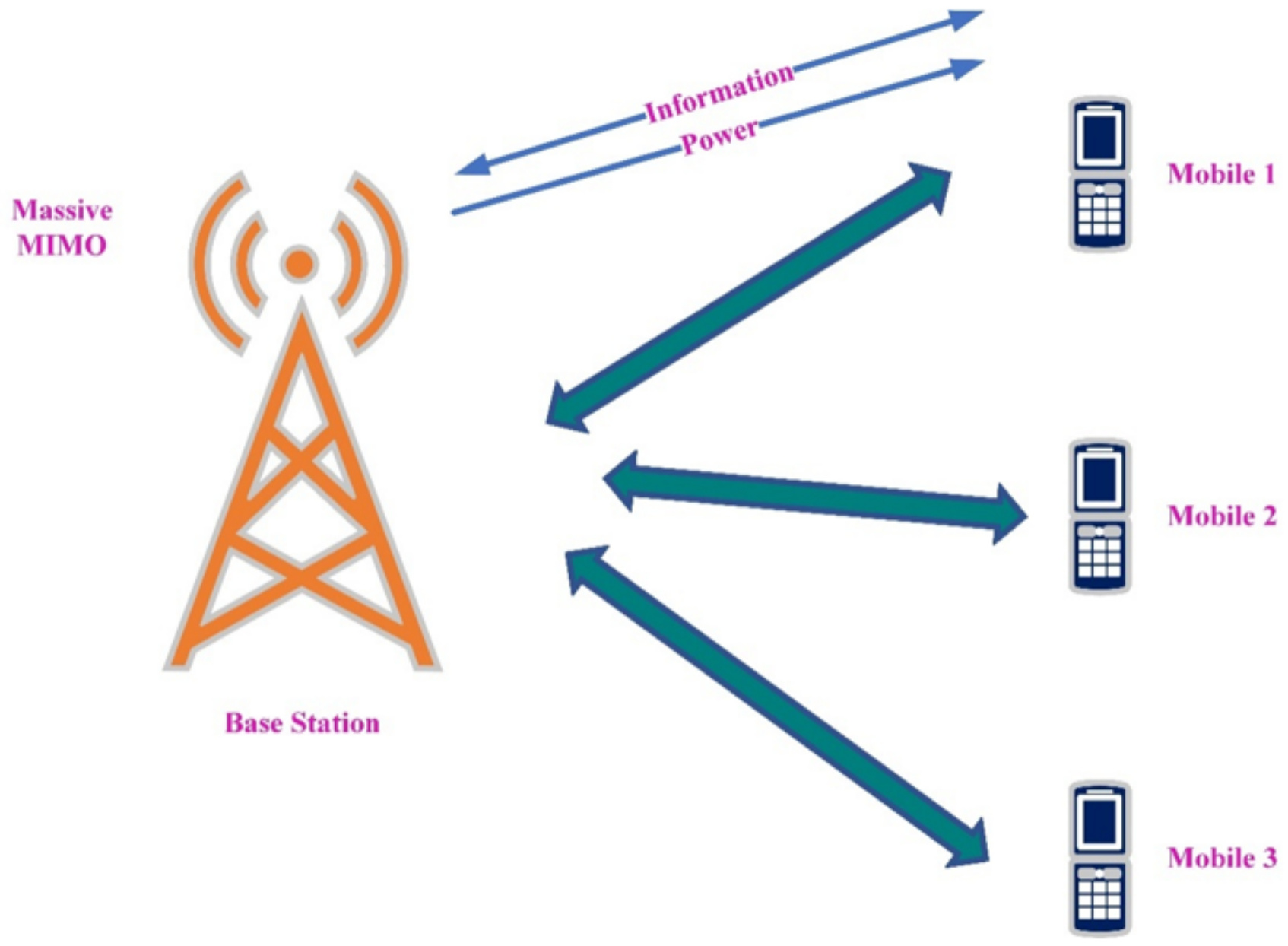

Figure 3

Power transfer 


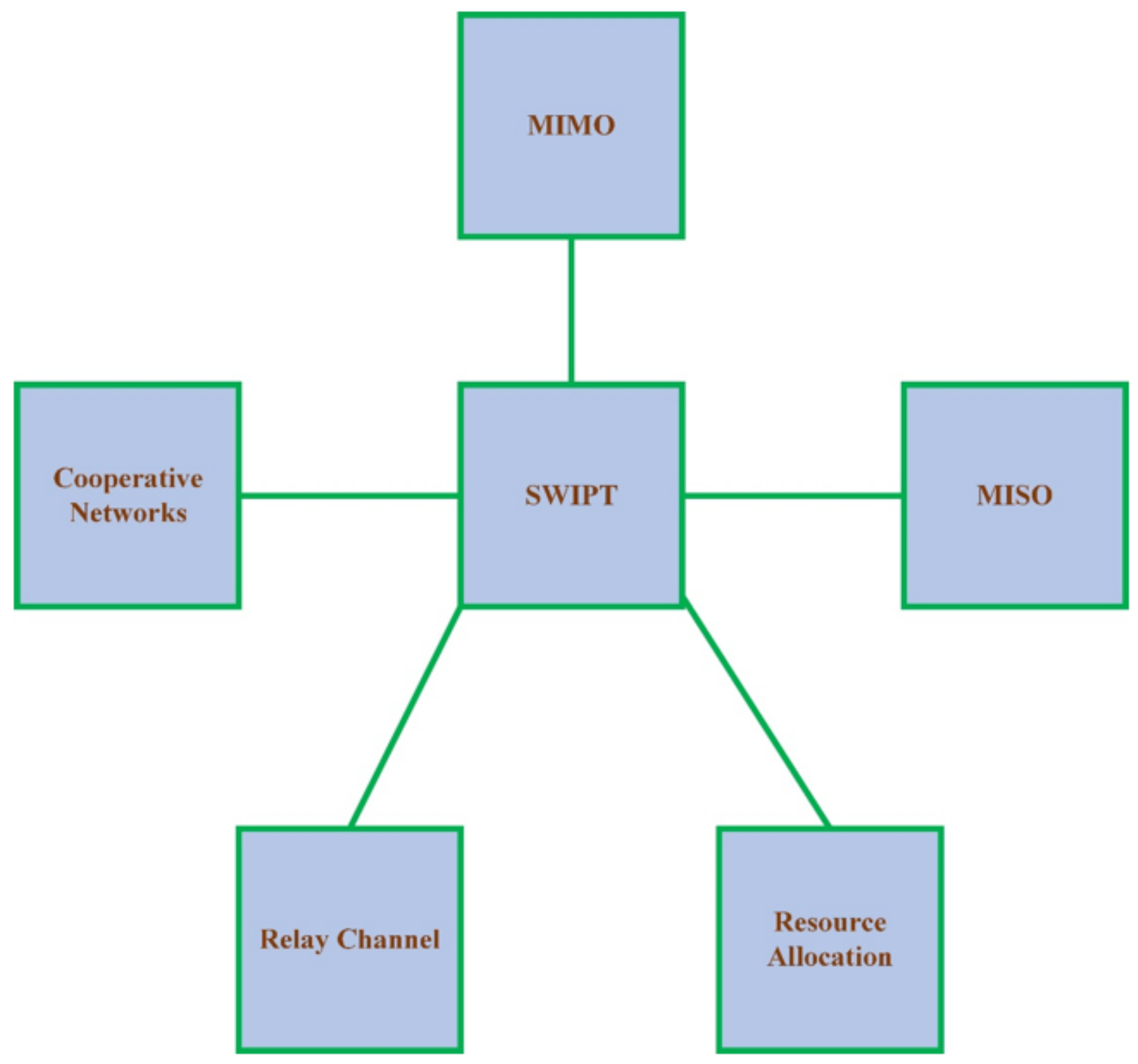

Figure 4

SWIPT based networks and channels 


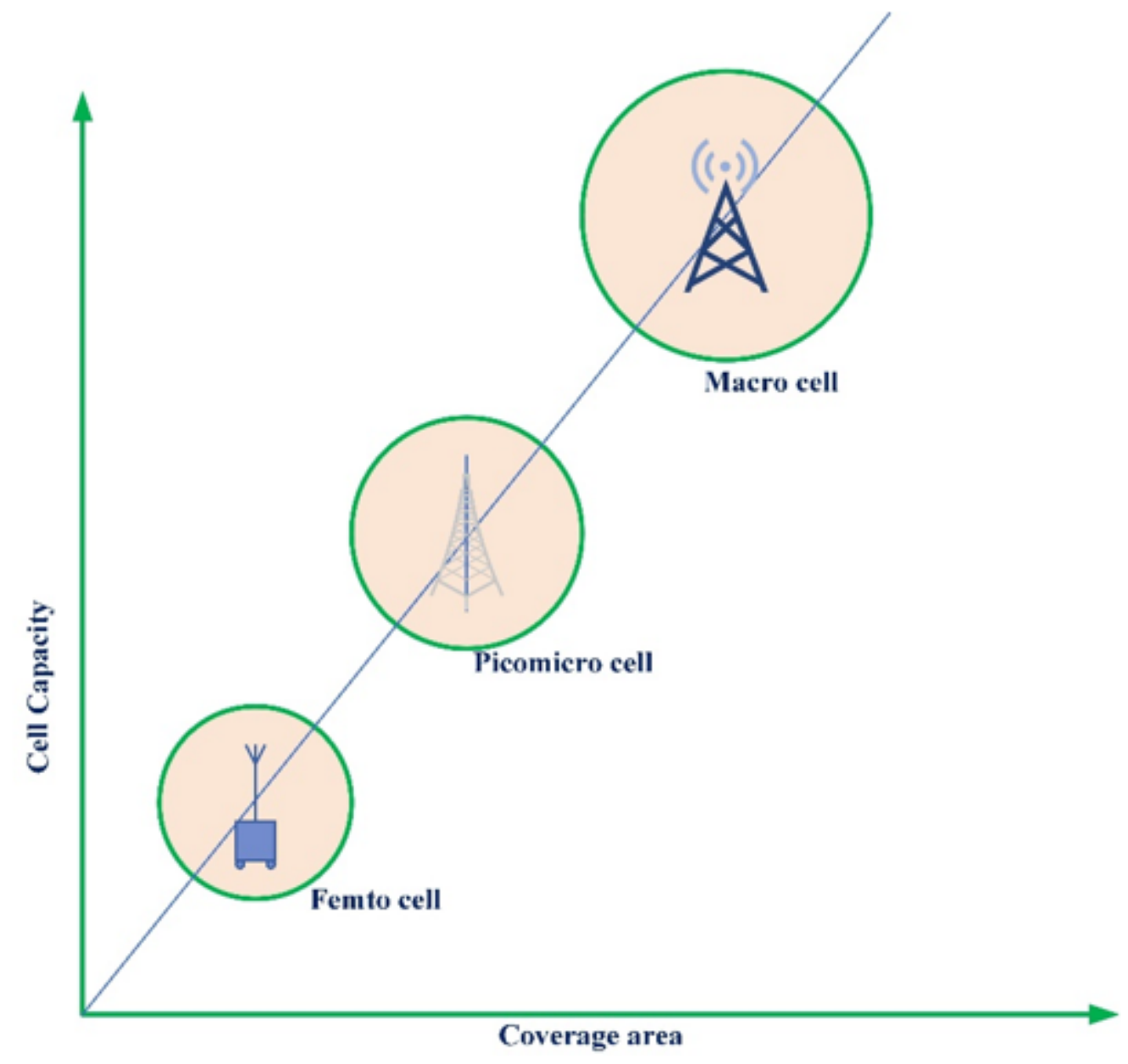

Figure 5

The capacity of cell Vs coverage area 


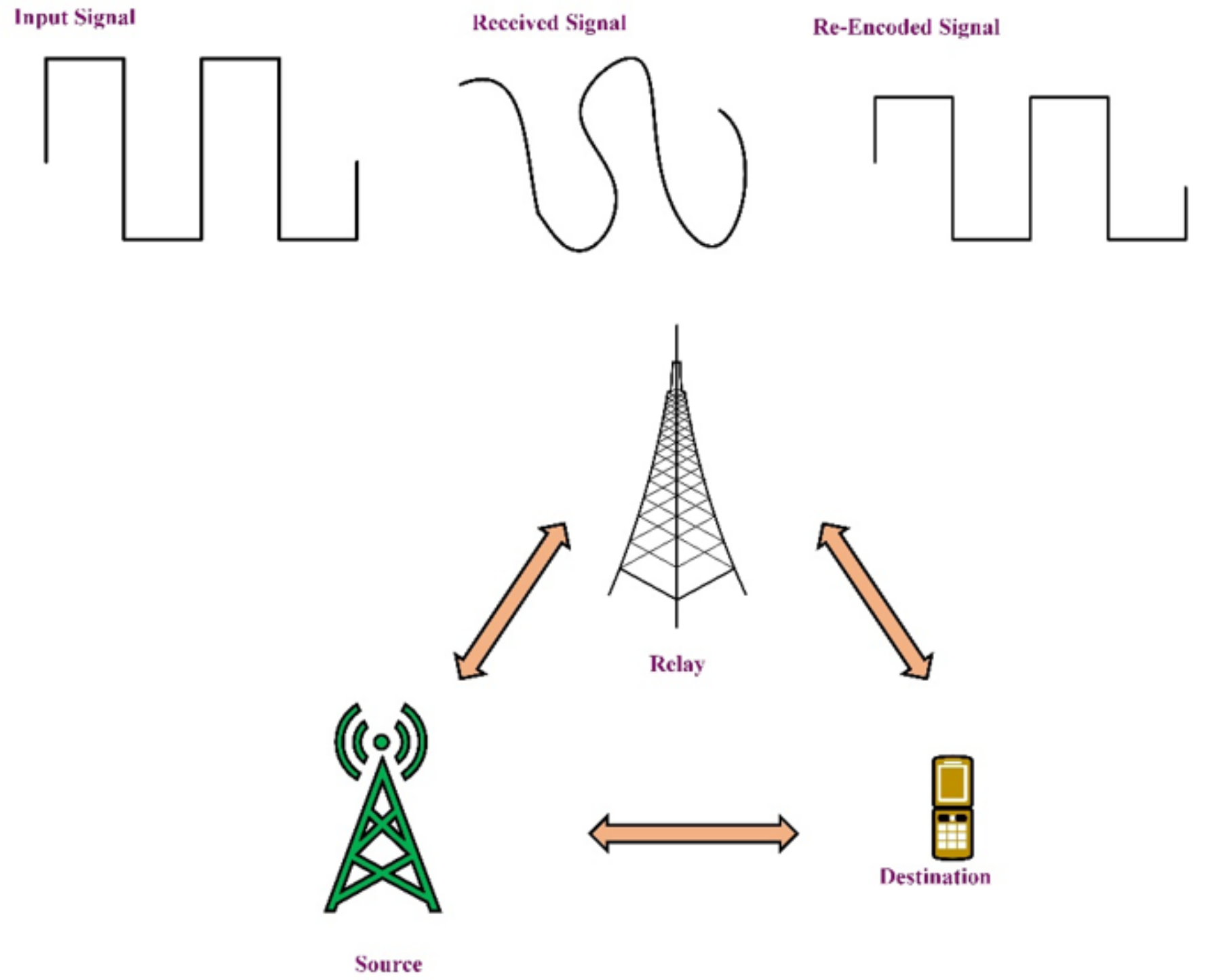

Figure 6

Decode and forward Relay 


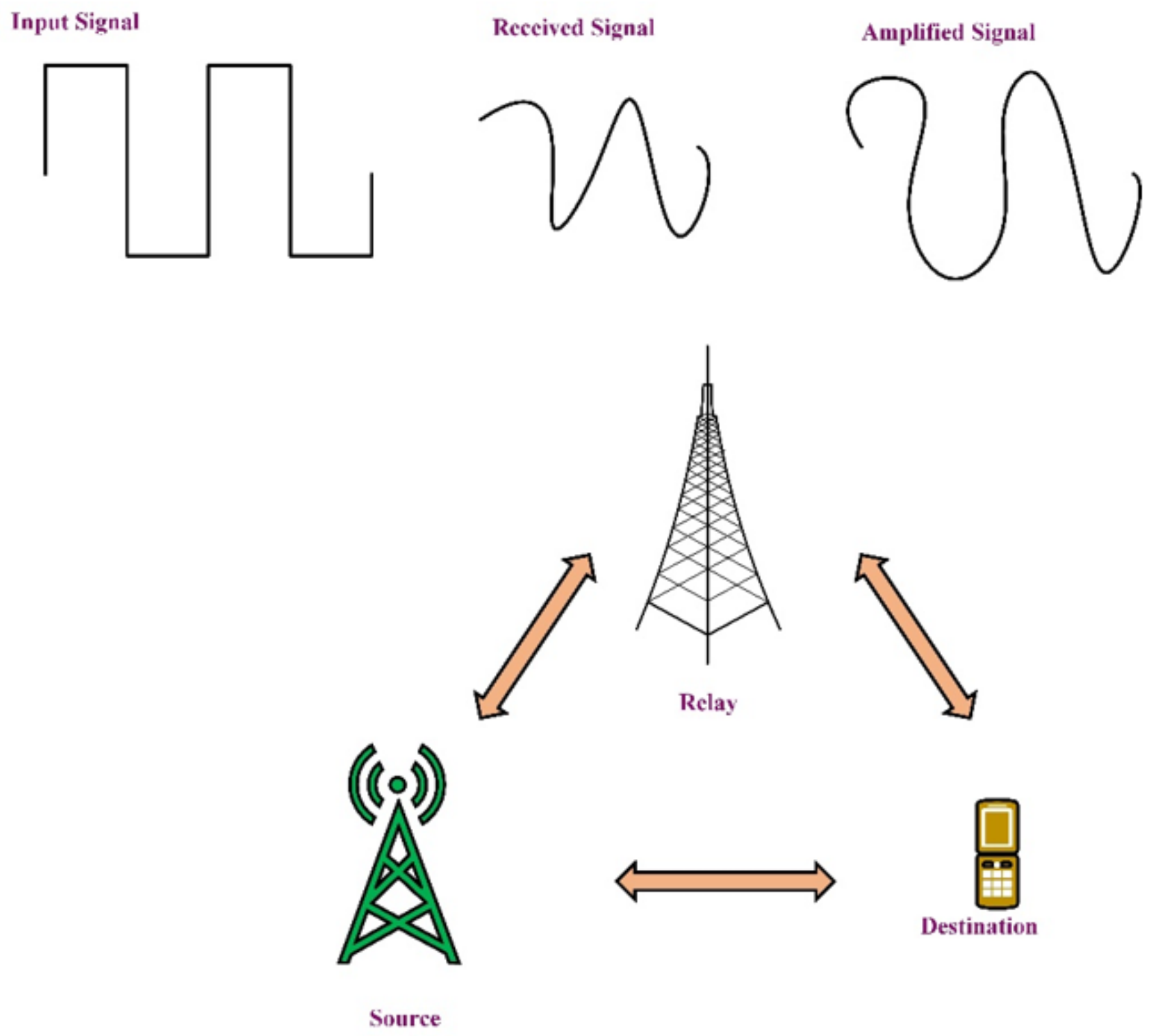

Figure 7

Amplify and forward Relay 


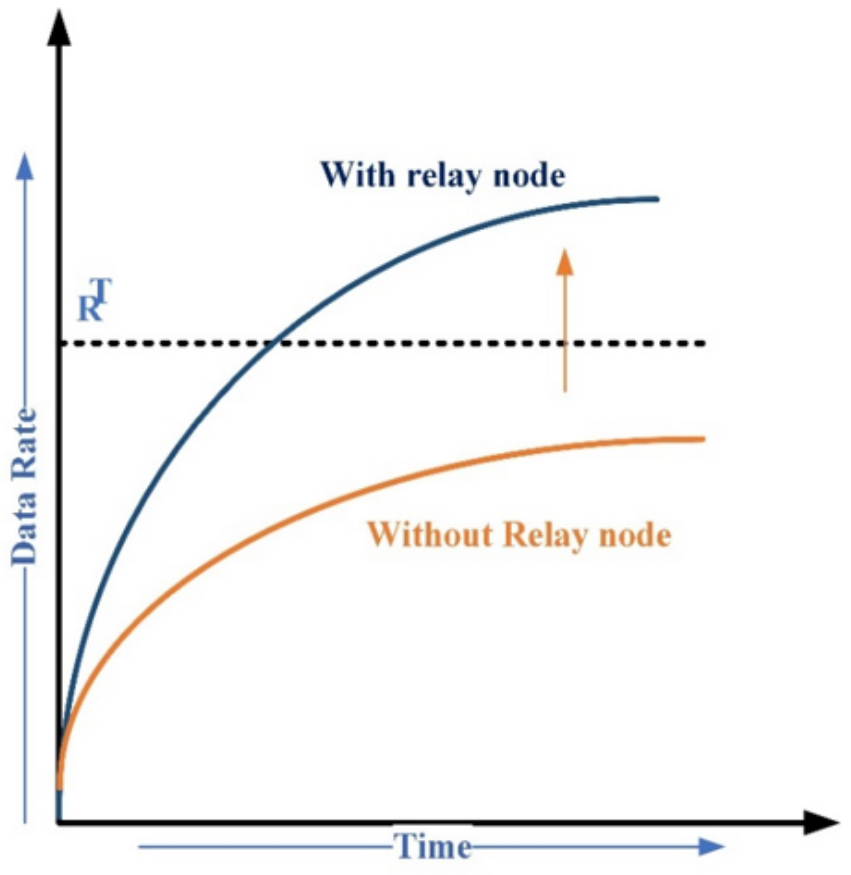

(a)

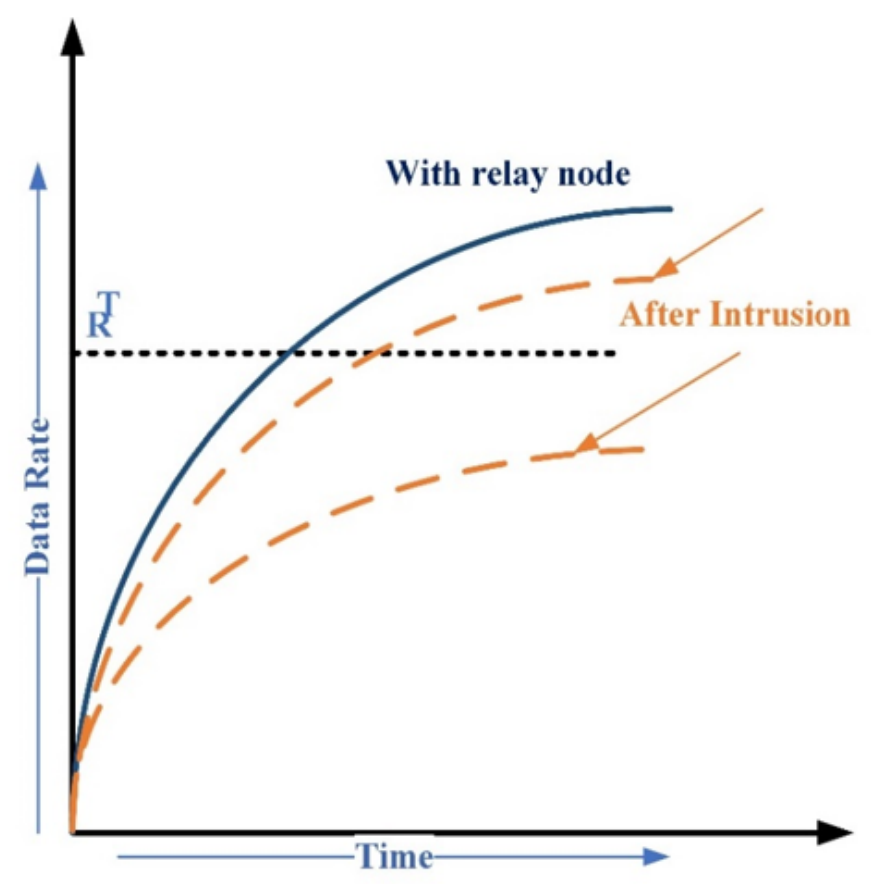

(b)

Figure 8

a) relay enhancement in data rate b) Intrusion impact on data rates
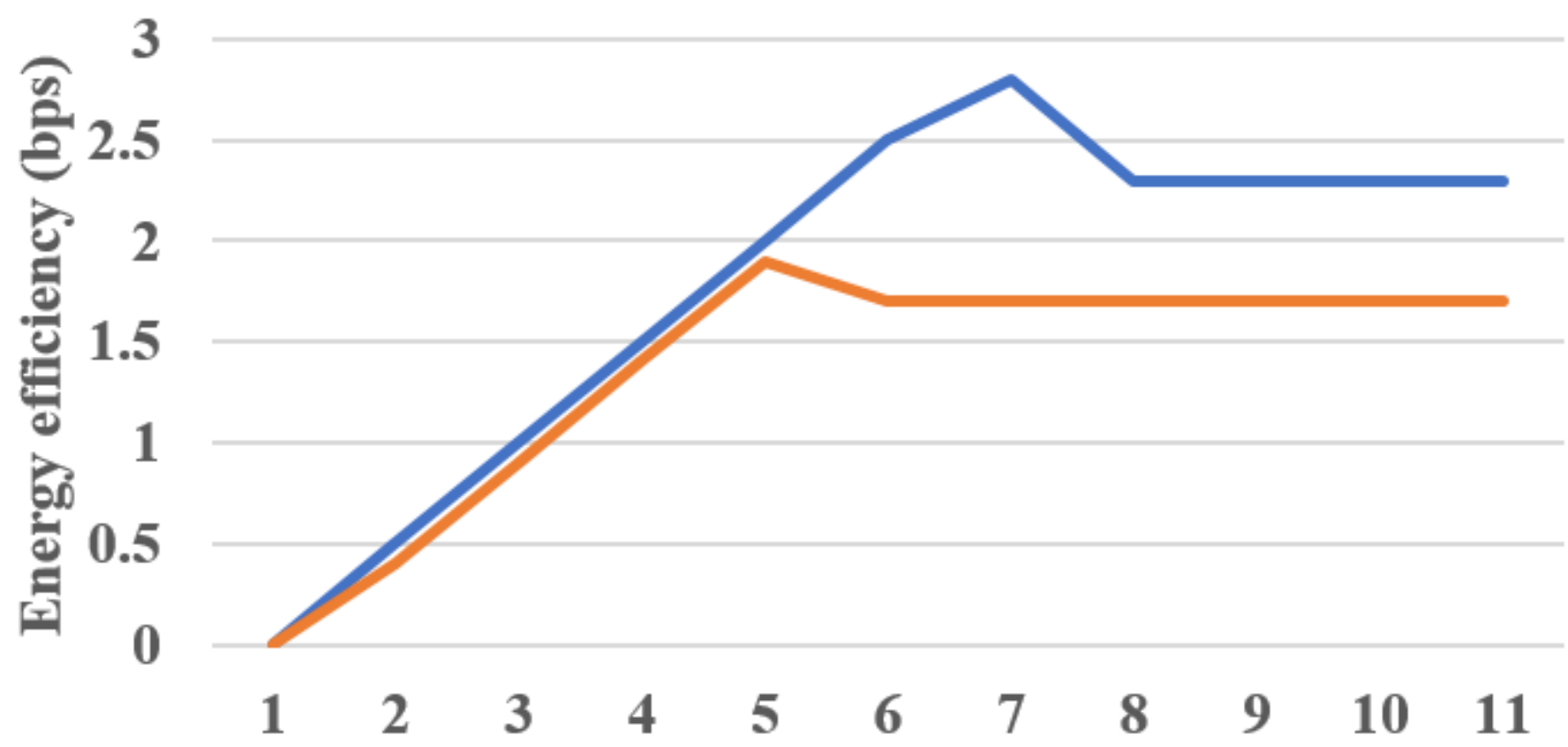

Index iteration 
Figure 9

Energy efficiency Vs number iteration

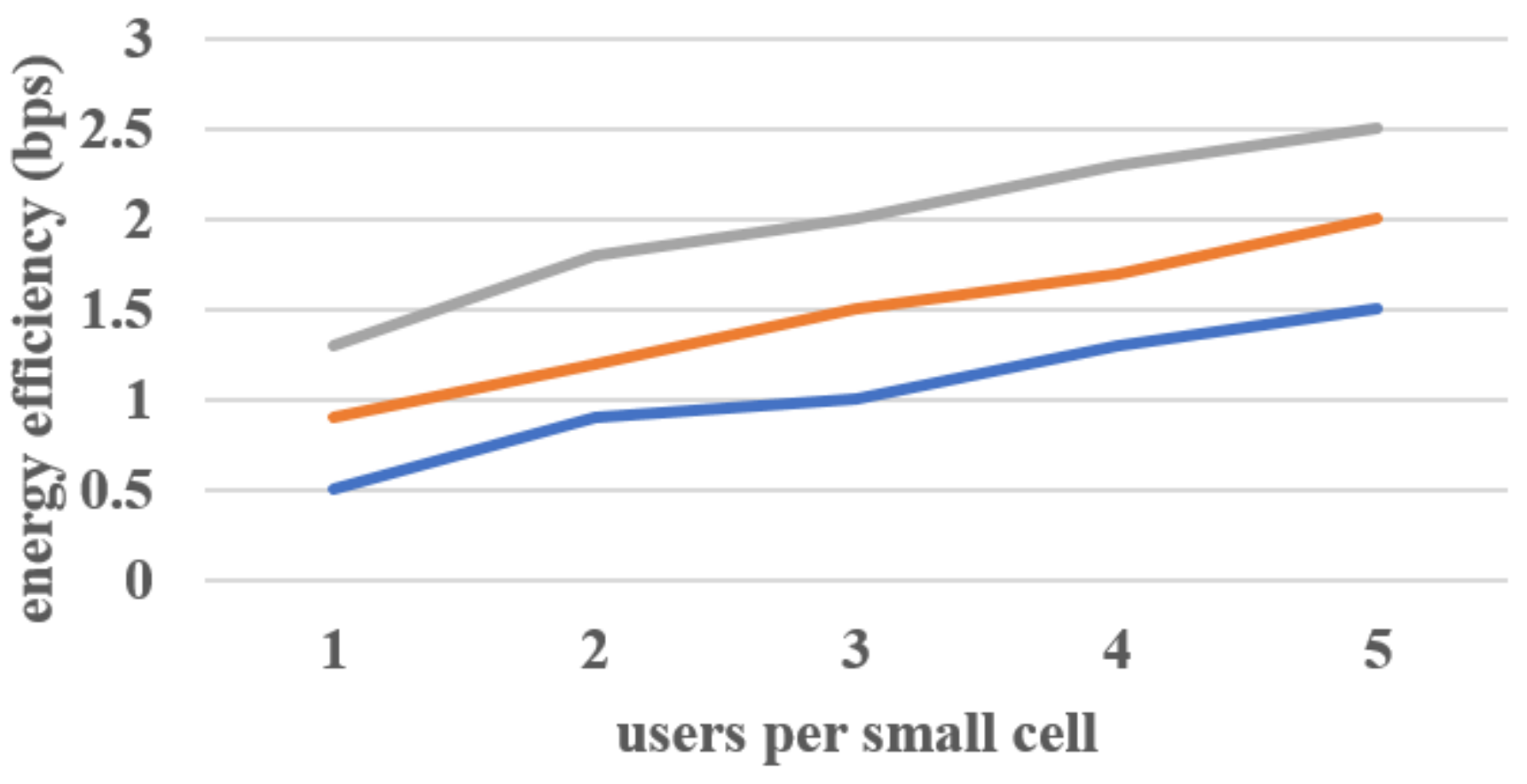

$\longrightarrow$ Series1 Series2 Series3

Figure 10

Energy efficiency Vs users' number per small cell 